Research Article

\title{
Macular Integrity Assessment and Fixation Analysis in Chronic Central Serous Chorioretinopathy
}

\author{
Joanna Dolar-Szczasny (D), Anna Święch-Zubilewicz, and Jerzy Mackiewicz \\ Department of Retina and Vitreous Surgery, Medical University of Lublin, Lublin, Poland \\ Correspondence should be addressed to Joanna Dolar-Szczasny; joannaszczasny@op.pl
}

Received 20 October 2017; Revised 13 January 2018; Accepted 5 February 2018; Published 14 March 2018

Academic Editor: Jakub J. Kaluzny

Copyright (c) 2018 Joanna Dolar-Szczasny et al. This is an open access article distributed under the Creative Commons Attribution License, which permits unrestricted use, distribution, and reproduction in any medium, provided the original work is properly cited.

\begin{abstract}
Purpose. To investigate retinal sensitivity characteristics associated with morphologic changes in the eyes exhibiting chronic central serous chorioretinopathy (CSC), using macular integrity assessment (MAIA) microperimetry. Methods. A retrospective, crosssectional, observational study was constructed. The eyes of patients classified as chronic CSC, according to the onset of subjective symptoms with serous retinal detachment, as confirmed by optical coherence tomography examination, were included in the study. Retinal sensitivity and fixation were analyzed by performing microperimetry examinations using the MAIA instrument. Results. We reviewed microperimetry examinations of 15 eyes of 15 patients (age: $28-51$ years) with chronic CSC and mean best-corrected visual acuity of $-0.2 \operatorname{logMAR}$ units. The mean retinal integrity in the chronic CSC group was $49.0 \pm 27.6$, which was significantly different from the control eyes. The mean average threshold in the eyes with chronic CSC was $24.7 \pm 5.8 \mathrm{~dB}$, which also was significantly different from the control eyes. Fixation stability was significantly different between the CSC and control eyes for the P1 parameter $(90.1 \pm 13.7$ versus $99.3 \pm 1.5)$, and for the P2 parameter $(97.4 \pm 4.0$ versus $100.0 \pm 0.0)$. Conclusion. New microperimetry technology may provide valuable information regarding the visual status of chronic CSC cases. Our findings suggest that retinal sensitivity and fixation stability in chronic CSC eyes may serve as useful indicators for assessing the effectiveness of clinical treatments.
\end{abstract}

\section{Introduction}

Central serous chorioretinopathy (CSC) is a macular disease that typically affects young and middle-aged adults. It is characterized by a serous retinal detachment at the posterior pole. Patients complain of blurred vision in the central or paracentral visual field and varying degrees of metamorphopsia, micropsia, central scotoma, and low-contrast sensitivity. In the majority of patients, CSC is self-limiting, and visual acuity recovers fully after subretinal fluid reabsorption [1]. However, in some patients, serous retinal detachment does not resolve for many months; even those with good visual acuity can experience a reduction in other visual functions, for example, contrast sensitivity, color discrimination, dark adaptation, focal macular electroretinograms, and macular sensitivity. In a small percentage of patients with CSC, prolonged recovery and recurrent episodes may lead to severe visual loss [2-8].
CSC is likely caused by choroidal vascular hyperpermeability that can be visualized on fluorescein angiography (FA) by focal leakage at the level of the retinal pigment epithelium (RPE) [9]. Some patients may present with multiple pinpoint leaks in FA or with a smokestack fluorescein pattern. FA may also show evidence of previous CSC episodes limited to the extramacular area; these may go undetected, as they are asymptomatic. In patients with long-standing CSC, findings may include focal or diffuse RPE atrophy and areas of RPE pigment clumping. Other notable findings include evidence of gravity-driven descending tracts of subretinal fluid on FA or fundus autofluorescence (FAF) images. These tracts are initially hyperautofluorescent in an acute phase of the disease, then become increasingly hypoautofluorescent as RPE cells are damaged within the area of fluid leakage. The staining of the inner choroid within the macular region and in the periphery, as seen on mid-phase indocyanine green (ICG) angiography, is the primary evidence of 
choroidal hyperpermeability. Focal or diffuse RPE defects in cases of chronic CSC are best visualized in FAF images, which show hypofluorescence spots in the damaged area. Subretinal fluid presence, due to neurosensory retinal detachment, can be visualized and monitored by noninvasive optical coherence tomography (OCT) [10]. Enhanced depth imaging spectral-domain optical coherence tomography (SD-OCT) findings demonstrate a very thick choroid in both eyes of patients with CSC [11]. The acute phase of CSC demonstrates a self-limiting, natural outcome; in contrast, the chronic form of CSC - with sustained subretinal fluid-may cause permanent visual disturbances. Therefore, analyzing functional parameters of the chronic form of CSC is important for both timing of treatment and prediction of prognosis.

Typically, CSC is classified as chronic through subjective patient history, and there has not yet been a definite, objective method to estimate the extent of subretinal fluid that might cause retinal dysfunction. Microperimetry is a very advanced technique for evaluating retinal sensitivity. Currently, it is considered a useful tool in many ophthalmologic clinical trials; notably, 78 clinical trials, in the clinical trial registry of the United States National Institutes of Health, use microperimetry evaluation-five of these involve CSC. A better understanding of the chronicity of CSC is important in determining treatment options; thus, we analyzed the retinal sensitivity of patients with chronic CSC, according to the onset of subjective symptoms, using macular integrity assessment (MAIA) microperimetry.

MAIA is a third-generation fundus perimeter, which permits differentiation between normal age-related loss of macular sensitivity and pathological (disease state) changes that require treatment. It provides a first step in retinal disease management, thereby enabling clinicians to test macular function and follow sequential changes in retinal disease progression. This will improve diagnosis and treatment of patients by permitting the detection of disease changes at an earlier stage than can be achieved using conventional testing methods. In this study, we aimed to present retinal sensitivity characteristics associated with morphological changes in CSC, using MAIA microperimetry.

\section{Material and Methods}

We retrospectively reviewed medical records of patients with idiopathic CSC, who were examined at the Retinal and Vitreous Surgery Department of Medical University in Lublin, from December 2014 to June 2017. Idiopathic CSC was diagnosed based on the following: presence of a serous detachment of the neurosensory retina involving the macula, as demonstrated by SD-OCT; leakage at the level of RPE on FA; and indirect ophthalmoscopy. We analyzed the examinations of patients who were observed for $>6$ months where subretinal fluid was still present. Only the eyes that presented with subretinal fluid in the foveal area, on OCT, were included in this study. Exclusion criteria were corneal or lens opacities, glaucoma or ocular hypertension, and a history of posterior uveitis, retinal detachment, ocular trauma, or optic

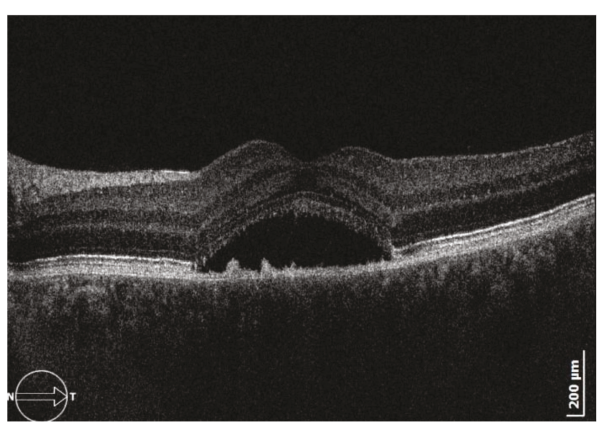

FIGURE 1: Optical coherence tomography of the macular region with subretinal fluid.

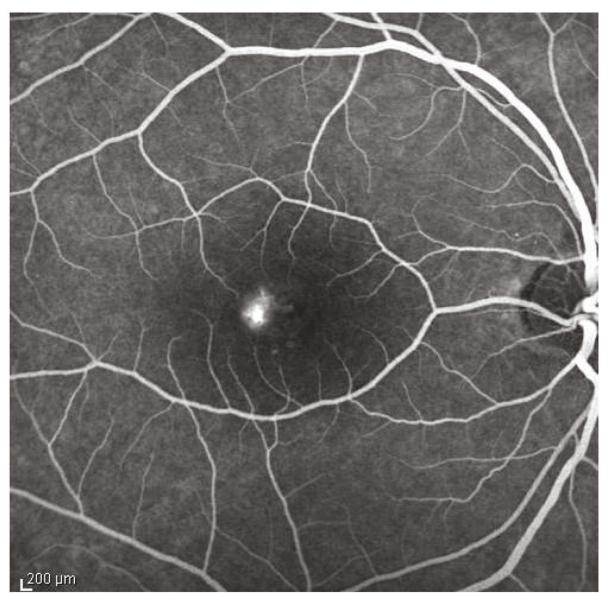

FIGURE 2: Fluorescein angiography of the eye with central serous chorioretinopathy.

neuropathy. None of the patients had been previously treated by laser photocoagulation or photodynamic therapy.

SD-OCT was performed using an SOCT Copernicus HR (OPTOPOL Technology, Poland) cube volume scan (Figure 1). FA and FAF images were obtained using an HRA2 confocal scanning laser ophthalmoscope (Heidelberg Retina Angiograph, Heidelberg, Germany) (Figure 2).

In addition to undergoing a comprehensive ophthalmologic examination-including best-corrected visual acuity (BCVA) measurement with EDTRS charts, slit lamp biomicroscopy, and indirect ophthalmoscopy-all patients had undergone microperimetry examination.

Microperimetry examinations were performed using the MAIA (CenterVue, Padova, Italy) microperimeter (Figure 3).

All examinations and data collection were performed in accordance with the 2000 revision of the Declaration of Helsinki. Approval was obtained from the Institutional Review Board of Medical University in Lublin, Poland.

2.1. Microperimetry Examinations. Microperimetry testing was performed using standardized mesopic testing conditions. Patients were instructed about the procedure, and tests were conducted with the examiner viewing the fundus on the device monitor in real time, while the patient was shown test stimuli. The examination was performed in a darkened and quiet room, following pupil dilatation. 


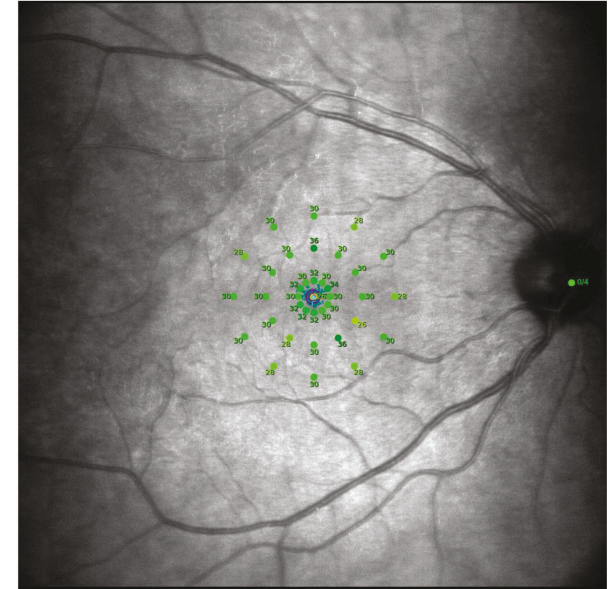

FIGURE 3: Microperimetry examination of the eye with central serous chorioretinopathy performed with the macular integrity assessment instrument.

The following parameters were used in the test: a 37stimulus grid overlying the central $10^{\circ}$, Goldmann III stimulus with a duration of $200 \mathrm{~ms}, 4-2$ threshold strategy, and a $1^{\circ}$ diameter red-circle fixation target. The standardized stimulus grid was composed of a single central foveal response and three concentric rings of retinal loci at $2^{\circ}, 6^{\circ}$, and $10^{\circ}$ from the center. Average sensitivity values for the macular region were calculated on the basis of the total projected stimulus mean value $\left(0-10^{\circ}\right.$ from the fixation point) and are presented as an average threshold value in decibels (dB). MAIA 4-2 follows the perimetric standard: it changes light intensity in 4 $\mathrm{dB}$ steps until there is a change from not seen to seen stimuli (or from seen to not seen). Then, it changes the intensity in 2 $\mathrm{dB}$ steps until the stimulus is not seen again (or seen again). The standard MAIA examination, using the 4-2 strategy, has an average duration of 5.5 minutes.

A unique parameter of the MAIA microperimeter is the macular integrity index, which is a proprietary statistical value that is calculated by use of a neural network multivariate model (the EYEdBTM). The model includes age, average threshold value, a measurement of points with threshold $<25 \mathrm{~dB}$, and all measured threshold values; it is derived by comparison with the manufacturer's normative data and describes the likelihood that threshold values will differ significantly from normal values. The algorithm of the macular integrity index calculation is not published. The macular integrity index is a numerical value that describes the likelihood that a patient's responses are normal, suspect, or abnormal, when compared with age-adjusted normative data. MAIA automatically generates a macular integrity index, which is calculated using the number of stimuli lower than $25 \mathrm{~dB}$, the sensitivity of the central stimuli, and the fixation stability factor. Alone, the macular integrity index does not represent the severity of the disease process. Higher numbers suggest a greater likelihood of pathological findings, while lower values suggest a greater likelihood of normal findings. Macular integrity index reflects the functional status of the eye with macular disorder, revealing morphological alterations in the macular region. There is no direct relationship between the average threshold value and the macular integrity index. In fact, it is possible for the average threshold value to be normal in a test subject who exhibits an abnormal macular integrity index. Notably, the macular integrity index is only present in examinations that are performed using the standard MAIA stimuli grid and the 4-2 projection strategy.

Fixation stability was assessed by tracking eye movements 25 times/sec and plotting the resulting distribution over the scanning laser ophthalmoscope image. Fixation characteristics were calculated automatically by the MAIA microperimetry software, after a landmark had been located in the center of the fovea. Fixation stability (P1 and P2) was measured by calculating the percentage of fixation points (\%) located within a distance of $1^{\circ}$ and $2^{\circ}$, respectively. Automatic classification of stability was based on the following criteria: (1) if $>75 \%$ of the fixation points were located within P1, fixation was classified as "stable"; (2) if $<75 \%$ of the fixation points were located within P1, but $>75 \%$ of the fixation points were located within P2, fixation was classified as "relatively unstable"; and (3) if $<75 \%$ of the fixation points were located within P2, the fixation was classified as "unstable" [12].

2.2. Data Analyses. Data are presented as mean values with standard deviation $( \pm \mathrm{SD})$. Results from age-matched control eyes (age range, $24-47$ years; mean age, 39 years) and eyes with chronic CSC were compared by Student's $t$ test.

All statistical analyses were performed using STATISTICA 12 statistical software (StatSoft Polska, Krakow, Poland). Statistical significance was defined as a $p$ value $<0.05$.

\section{Results}

A total of 15 eyes of 15 patients with chronic CSC were included in this study. The mean age of the patients in the study was 40.6 years (range, 28-51 years; median age, 41 years). A total of 13 patients (86.7\%) were men, and two patients (13.3\%) were women. Ten of 15 patients exhibited focal hypofluorescence changes within FAF images. Because of poor fixation stability in patient number 1, we excluded the patient's data from our statistical analysis. Detailed clinical profiles of chronic CSC patients and control subjects are presented in Tables 1 and 2.

In the eyes of CSC patients, the mean BCVA at the time of microperimetry examinations was $0.2 \log \mathrm{MAR}$ units (range: $0.7-0.0$; median: $-0.2 \pm 0.2$ ), with a statistically significant difference between CSC eyes and control eyes. The mean retinal integrity index in the eyes of CSC patients was $49.0 \pm 27.6$, which was statistically different from the control eyes $(2.6 \pm 1.6)(p<0.05)$. The mean average threshold in the eyes of CSC patients was $24.7 \pm 5.8 \mathrm{~dB}$, which was statistically significantly lower than that in the control eyes $(31.4 \pm 2.7 \mathrm{~dB})(p<0.05)$. Fixation stability in most cases was classified as stable but was statistically different between P1 (CSC eyes: $90.1 \pm 13.7$ versus control eyes: $99.3 \pm 1.5$ ) and P2 (CSC eyes: $97.4 \pm 4.0$ versus control eyes: $100.0 \pm 0.0$ ) parameters (Table 3 ). 
TABLE 1: Clinical characteristics of patients with chronic central serous chorioretinopathy.

\begin{tabular}{|c|c|c|c|c|c|c|}
\hline Patient number & $\begin{array}{c}\text { Age } \\
\text { (years) }\end{array}$ & $\begin{array}{l}\text { Visual acuity } \\
\text { (logMAR) }\end{array}$ & $\begin{array}{l}\text { Macular } \\
\text { integrity }\end{array}$ & $\begin{array}{c}\text { Average } \\
\text { threshold }(\mathrm{dB})\end{array}$ & $\begin{array}{l}\text { Microperimetry fixation } \\
\text { stability P1 (\%) }\end{array}$ & $\begin{array}{c}\text { Microperimetry fixation } \\
\text { stability P2 (\%) }\end{array}$ \\
\hline 2 & 40 & 0.18 & 48.5 & 27.3 & 100 & 100 \\
\hline 3 & 35 & 0.0 & 13.7 & 29.0 & 94 & 99 \\
\hline 4 & 43 & 0.1 & 53.4 & 27.7 & 72 & 90 \\
\hline 5 & 36 & 0.2 & 93.6 & 25.5 & 100 & 100 \\
\hline 6 & 43 & 0.7 & 52.6 & 12.1 & 76 & 95 \\
\hline 7 & 47 & 0.1 & 35.0 & 28.6 & 99 & 100 \\
\hline 8 & 40 & 0.18 & 92.0 & 26.1 & 100 & 100 \\
\hline 9 & 35 & 0.18 & 7.6 & 29.4 & 82 & 97 \\
\hline 10 & 48 & 0.3 & 58.4 & 22.5 & 100 & 100 \\
\hline 11 & 36 & 0.2 & 41.6 & 28.1 & 94 & 100 \\
\hline 12 & 41 & 0.5 & 65.6 & 13.7 & 90 & 95 \\
\hline 13 & 42 & 0.3 & 81.4 & 16.5 & 56 & 88 \\
\hline 14 & 45 & 0.2 & 56.4 & 27.3 & 99 & 100 \\
\hline 15 & 51 & 0.2 & 26.9 & 27.5 & 100 & 100 \\
\hline
\end{tabular}

TABLE 2: Clinical characteristics of control subjects.

\begin{tabular}{|c|c|c|c|c|c|c|}
\hline Patient number & $\begin{array}{c}\text { Age } \\
\text { (years) }\end{array}$ & $\begin{array}{l}\text { Visual acuity } \\
\text { (logMAR) }\end{array}$ & $\begin{array}{l}\text { Macular } \\
\text { integrity }\end{array}$ & $\begin{array}{c}\text { Average } \\
\text { threshold }(\mathrm{dB})\end{array}$ & $\begin{array}{l}\text { Microperimetry fixation } \\
\text { stability P1 (\%) }\end{array}$ & $\begin{array}{c}\text { Microperimetry fixation } \\
\text { stability P2 (\%) }\end{array}$ \\
\hline 1 & 36 & 0.0 & 3.6 & 30.2 & 99 & 100 \\
\hline 2 & 35 & 0.0 & 0.2 & 35.5 & 100 & 100 \\
\hline 3 & 42 & 0.0 & 1.4 & 30.8 & 100 & 100 \\
\hline 4 & 43 & 0.0 & 2.8 & 28.2 & 100 & 100 \\
\hline 5 & 35 & 0.0 & 1.2 & 29.6 & 100 & 100 \\
\hline 6 & 38 & 0.0 & 5.0 & 32.7 & 100 & 100 \\
\hline 7 & 24 & 0.0 & 3.8 & 31.8 & 98 & 100 \\
\hline 8 & 41 & 0.0 & 4.2 & 27.8 & 100 & 100 \\
\hline 9 & 43 & 0.0 & 2.4 & 33.8 & 97 & 100 \\
\hline 10 & 47 & 0.1 & 1.2 & 29.6 & 100 & 100 \\
\hline 11 & 40 & 0.0 & 5.6 & 34.2 & 95 & 100 \\
\hline 12 & 39 & 0.0 & 2.2 & 35.2 & 100 & 100 \\
\hline 13 & 37 & 0.0 & 1.8 & 32.8 & 100 & 100 \\
\hline 14 & 46 & 0.1 & 0.4 & 31.6 & 100 & 100 \\
\hline 15 & 42 & 0.0 & 3.4 & 26.8 & 100 & 100 \\
\hline
\end{tabular}

TABLE 3: Mean visual acuity, macular integrity index, average threshold, and fixation stability in eyes with chronic central serous chorioretinopathy and control eyes.

\begin{tabular}{|c|c|c|c|c|}
\hline & Control eyes $($ mean $\pm \mathrm{SD})$ & Eyes with chronic CSC (mean $\pm \mathrm{SD})$ & Statistic ( $t$ test) & $p$ value \\
\hline Visual acuity (logMAR) & $0.0 \pm 0.0$ & $0.2 \pm 0.2$ & 0.00 & $p<0.05$ \\
\hline Macular integrity & $2.6 \pm 1.6$ & $49.0 \pm 27.6$ & 0.00 & $p<0.05$ \\
\hline Average threshold $(\mathrm{dB})$ & $31.4 \pm 2.7$ & $24.7 \pm 5.8$ & 0.01 & $p<0.05$ \\
\hline Fixation stability P1 (\%) & $99.3 \pm 1.5$ & $90.1 \pm 13.7$ & 0.01 & $p<0.05$ \\
\hline Fixation stability P2 (\%) & $100.0 \pm 0.0$ & $97.4 \pm 4.0$ & 0.02 & $p<0.05$ \\
\hline
\end{tabular}

\section{Discussion}

When evaluating patients with macular diseases, measurement of retinal sensitivity and fixation stability by microperimetry is likely to provide a more precise examination than determination of simple visual acuity [13], as visual acuity is measured using high-contrast optotypes under bright light conditions, and, therefore, does 
not fully represent the visual functions of patients in their daily lives.

The MAIA microperimeter is a relatively new instrument that couples digital fundus imaging with automated microperimetry. To our knowledge, this study is the first to report both examination of patients with chronic CSC and analysis of the macular integrity index, using the MAIA microperimeter. Another study that used MAIA to examine patients with acute CSC reported that microperimetry of $<20 \mathrm{~dB}$ had a relative risk of 4.5 for development of subretinal fluid persistence [14]. All microperimetry tests were performed with dilated pupils, as pupil dilatation does not affect the test results.

CSC is regarded as a self-limiting disease, with good prognosis after subretinal fluid resolution. However, in chronic cases, persistent serous detachment of the macular region may cause RPE damage and permanent visual dysfunction [15]. Recent microperimetry studies have shown that eyes with resolved CSC may exhibit significantly lower central retinal sensitivity, even after achievement of good central visual acuity $[16,17]$. In contrast, we analyzed patients who exhibited presence of chronic subretinal fluid. Our analysis shows that the eyes with chronic CSC can exhibit lower macular sensitivity values in the central macula, compared with the control eyes; this is consistent with observations of decreased VA. There was also statistical evidence that the index of macular integrity was different between the CSC eyes and control eyes; however, this measurement does not reflect the severity of CSC pathology and has no correlation with average threshold values. Finally, fixation parameters were worse not only for the central point of fixation (P1) in the chronic CSC eyes. P2 parameters were also outside normal limits, indicating that fixation stability in central and paracentral area was impaired.

Our study has several limitations, including a relatively small sample size and retrospective nature. Additionally, we could not correlate reductions in retinal sensitivity with patients' subjective symptoms. However, our study is notable for reporting parameters of macular dysfunction, in cases of chronic subretinal fluid presence, as part of natural history of the disease, which contrasts with studies that have reported posttreatment microperimetry results in the chronic CSC eyes [18-22]. Critically, prospective studies, with a larger number of cases, are required to confirm the applicability of these results.

\section{Conclusion}

Our findings are important in the determination of retinal sensitivity and fixation stability in the chronic CSC eyes that have experienced extended exposure to subretinal fluid. These parameters may be useful indicators for assessing the effectiveness of clinical treatments.

\section{Conflicts of Interest}

The authors declare that they have no conflicts of interest.

\section{References}

[1] M. Wang, I. C. Munch, P. W. Hasler, C. Prünte, and M. Larsen, "Central serous chorioretinopathy," Acta Ophthalmologica, vol. 86, no. 2, pp. 126-145, 2008.

[2] J. C. Folk, H. S. Thompson, D. P. Han, and C. K. Bron, "Visual function abnormalities in central serous retinopathy," Archives of Ophthalmology, vol. 102, no. 9, pp. 1299-1302, 1984.

[3] E. L. Chuang, D. M. Sharp, F. W. Fitzke, C. M. Kemp, A. L. Holden, and A. C. Bird, "Retinal dysfunction in central serous retinopathy," Eye, vol. 1, no. 1, pp. 120-125, 1987.

[4] Y. Miyake, N. Shiroyama, I. Ota, and M. Horiguchi, "Local macular electroretinographic responses in idiopathic central serous chorioretinopathy," American Journal of Ophthalmology, vol. 106, no. 5, pp. 546-550, 1988.

[5] M. Nagata and Y. Honda, "Macular ERG in central serous chorioretinopathy," Japanese Journal of Ophthalmology, vol. 15, pp. 9-16, 1971.

[6] M. F. Marmor and F. Tan, "Central serous chorioretinopathy: bilateral multifocal electroretinographic abnormalities," Archives of Ophthalmology, vol. 117, no. 2, pp. 184-188, 1999.

[7] T. S. Vajaranant, J. P. Szlyk, G. A. Fishman, J. P. Gieser, and W. Seiple, "Localized retinal dysfunction in central serous chorioretinopathy as measured using the multifocal electroretinogram," Ophthalmology, vol. 109, no. 7, pp. 1243-1250, 2002.

[8] T. Y. Lai, R. Y. Lai, J. W. Ngai, W. M. Chan, H. Li, and D. S. Lam, "First and second-order kernel multifocal electroretinography abnormalities in acute central serous chorioretinopathy," Documenta Ophthalmologica, vol. 116, no. 1, pp. 29-40, 2008.

[9] H. C. Gackle, G. E. Lang, K. A. Freissler, and G. K. Lang, "Clinical, fluorescein angiographic and demographic aspects in central serous chorioretinopathy," Der Ophthalmologe, vol. 95, no. 8, pp. 529-533, 1998.

[10] T. Iida, N. Hagimura, T. Sato, and S. Kisi, "Evaluation of central serous chorioretinopathy with optical coherence tomography," American Journal of Ophthalmology, vol. 129, no. 1, pp. 16-20, 2000.

[11] Y. Imamura, T. Fujiwara, R. Margolis, and R. F. Spaide, "Enhanced depth imaging optical coherence tomography of the choroid in central serous chorioretinopathy," Retina, vol. 29, no. 10, pp. 1469-1473, 2009.

[12] MAIA, "Macular integrity assessment operating manual," 2015, https://www.haag-streit.com/fileadmin/Haag-Streit_UK/ Downloads/Centervue_downloads/MAIA_downloads/MAIA_ Operating_Manual.pdf.

[13] K. Rohrschneider, S. Bultmann, and C. Sprinter, "Use of fundus perimetry (microperimetry) to quantify macular sensitivity," Progress in Retinal and Eye Research, vol. 27, no. 5, pp. 536-548, 2008.

[14] L. Roisman, J. C. Ribeiro, F. V. Fechine et al., "Does microperimetry have a prognostic value in central serous chorioretinopathy?" Retina, vol. 34, no. 4, pp. 713-718, 2014.

[15] M. S. Wang, B. Sander, and M. Larsen, "Retinal atrophy in idiopathic central serous chorioretinopathy," American Journal of Ophthalmology, vol. 133, no. 6, pp. 787-793, 2002.

[16] Y. Ojima, A. Tsujikawa, M. Hangai et al., "Retinal sensitivity measured with the micro perimeter 1 after resolution of central serous chorioretinopathy," American Journal of Ophthalmology, vol. 146, no. 1, pp. 77-84.e1, 2008. 
[17] H. Ozdemir, S. A. Karacorlu, F. Senturk, M. Karacorlu, and O. Uysal, "Assessment of macular function by microperimetry in unilateral resolved central serous chorioretinopathy," Eye, vol. 22, no. 2, pp. 204-208, 2008.

[18] K. Fujita, K. Shinoda, C. S. Matsumoto et al., "Microperimetric evaluation of chronic central serous chorioretinopathy after half-dose photodynamic therapy," Clinical Ophthalmology, vol. 6, pp. 1681-1687, 2012.

[19] K. Fujita, K. Shinoda, Y. Imamura et al., "Correlation of integrity of cone outer segment tips line with retinal sensitivity after half-dose photodynamic therapy for chronic central serous chorioretinopathy," American Journal of Ophthalmology, vol. 154, no. 3, pp. 579-585, 2012.

[20] R. Ehrlich, N. P. Mawer, C. H. Mody, C. S. Brand, and D. Squirrell, "Visual function following photodynamic therapy for central serous chorioretinopathy: a comparison of automated macular microperimetry versus best-corrected visual acuity," Clinical \& Experimental Ophthalmology, vol. 40, no. 1, pp. e32-e39, 2012.

[21] M. Reibaldi, F. Boscia, T. Avitabile et al., "Functional retinal changes measured by microperimetry in standard-fluence vs low-fluence photodynamic therapy in chronic central serous chorioretinopathy," American Journal of Ophthalmology, vol. 151, no. 6, pp. 953-960.e2, 2011.

[22] K. Fujita, M. Yuzawa, and R. Mori, "Retinal sensitivity after photodynamic therapy with half-dose verteporfin for chronic central serous chorioretinopathy: short-term results," Retina, vol. 31, no. 4, pp. 772-778, 2011. 


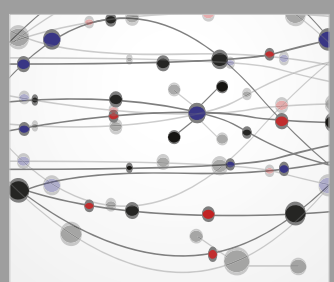

The Scientific World Journal
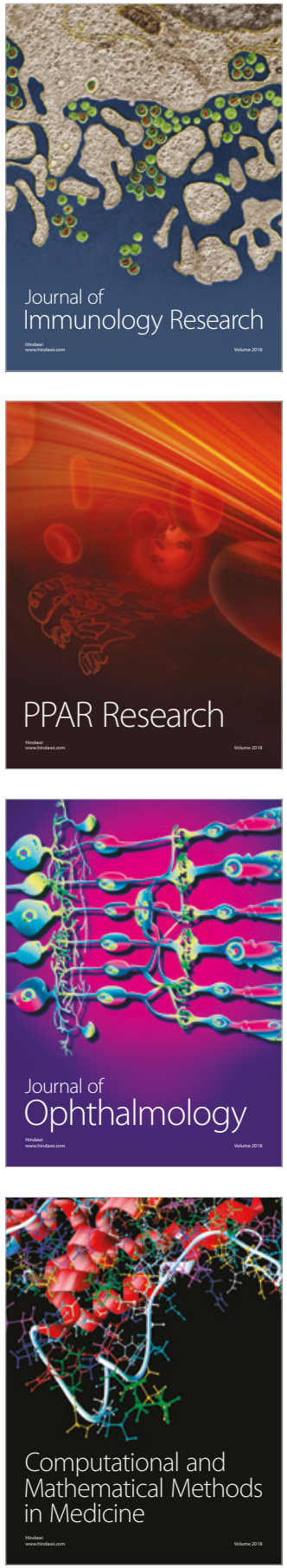

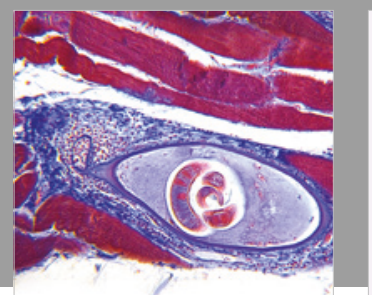

Gastroenterology Research and Practice

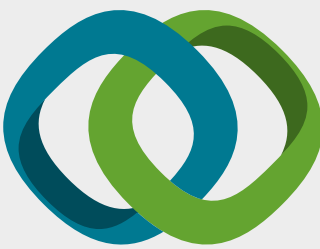

\section{Hindawi}

Submit your manuscripts at

www.hindawi.com
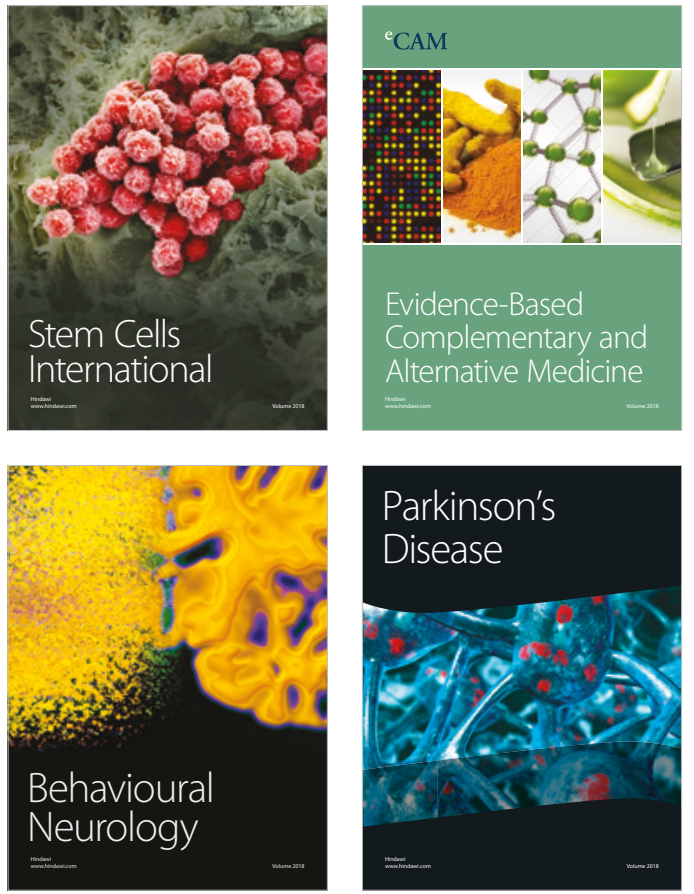

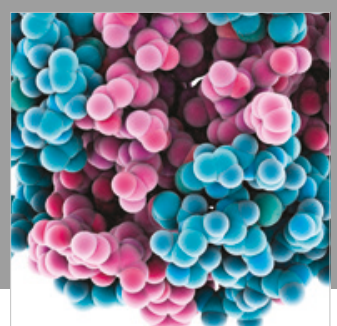

ournal of

Diabetes Research

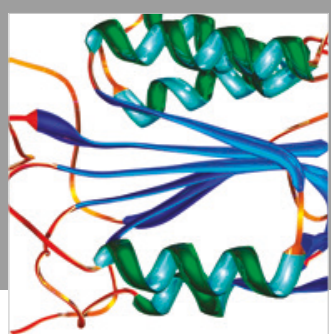

Disease Markers
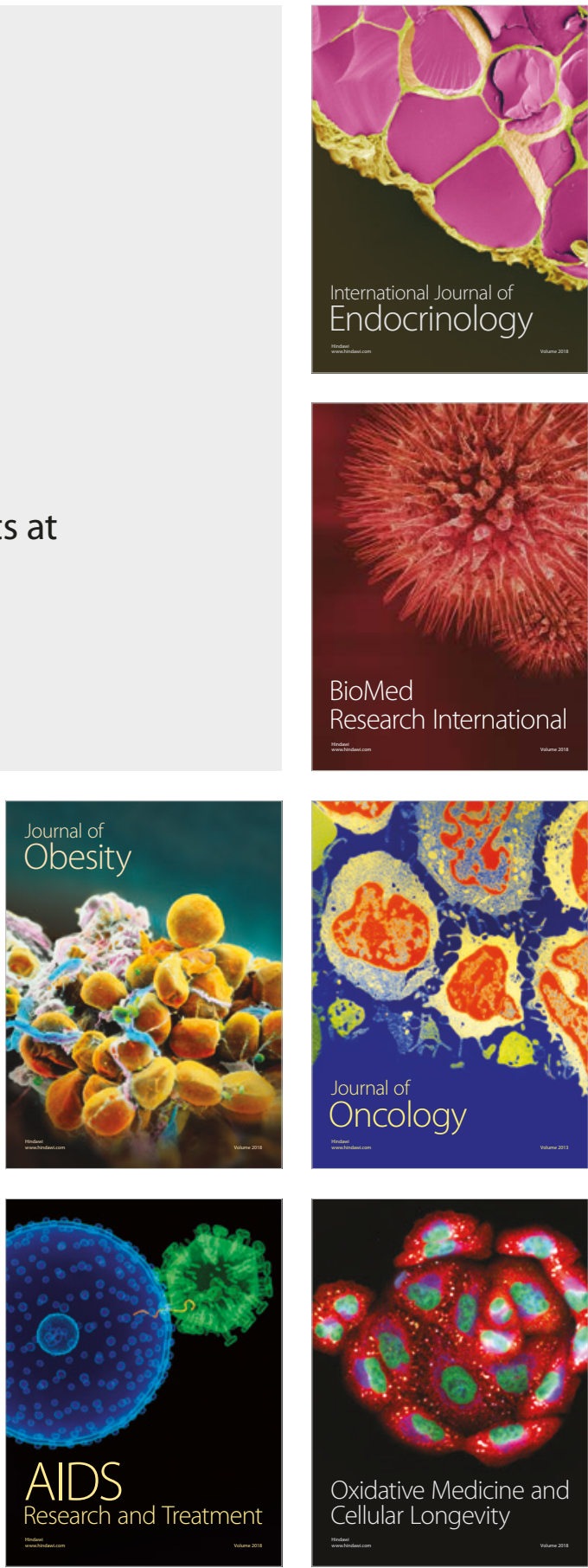